\title{
IMPACT OF MATERNAL DIABETES MELLITUS ON MORTALITY AND MORBIDITY OF PRETERM INFANTS (24-33 WEEKS GESTATION)
}

Y. Bental ${ }^{1,2}$, B. Reichman ${ }^{3,4}$, Y. Shiff ${ }^{5}$, M. Weisbrod ${ }^{5}$, V. Boyko ${ }^{3}$, F.B. Mimouni ${ }^{4,6}$, Israel Neonatal Network

${ }^{I}$ Neonatology, Laniado Hospital, Natanya, ${ }^{2}$ Technion, Israel Institute of Technology, The Ruth and Bruce Rappaport Faculty of Medicine, Haifa, ${ }^{3}$ Women and Children's Health Research Unit, Gertner Institute, TelHashomer, ${ }^{4}$ Sackler School of Medicine - Tel Aviv University, Tel Aviv, ${ }^{5}$ Department of Neonatology, Laniado Hospital, Natania, ${ }^{6}$ Pediatrics, Shaare Zedek Medical Center, Jerusalem, Israel

Objectives: To test the hypothesis that maternal DM increases the risk for mortality, RDS and major complications of prematurity.

Methods: Analysis of prospectively collected (1995-2007) Israel National VLBW infant database. Maternal DM was recorded as pre-gestational or gestational. Multivariable logistic regression analysis was used to assess the independent effect IDM status on mortality, RDS and other prematurity complications.

Results: Pregestational IDM's $(n=120)$ and gestational IDM's $(n=825)$ were very similar and pooled together for analyses. DM mothers were more likely to have received a complete course of prenatal steroids than control mothers. The IDM group had a slightly higher gestational age and birth-weight than the non IDM group. Distribution of birth weight percentiles and the mean birth weight z-scores were nearly identical. Apgar scores were statistically higher in IDM's than in controls. There were no significant differences between the two groups in terms of delivery room mortality, RDS, and other major complications of prematurity. Total mortality and BPD rates were significantly higher in the non diabetic group. The adjusted odds ratios for mortality, RDS, BPD at 28 days or 36 weeks postmenstrual age, IVH grades 3-4, PVL, ROP grades 3-4, NEC, and PDA were not significantly increased in the IDM group.

Conclusions: With modern management and adequate prenatal care, IDM's born VLBW do not appear to be at an excess risk of developing RDS or other major complications of prematurity as compared to non-IDM's infants. 